\title{
High Prevalence of Premalignant Lesions in Prophylactically Removed Breasts From Women at Hereditary Risk for Breast Cancer
}

\author{
By N. Hoogerbrugge, P. Bult, L.M. de Widt-Levert, L.V. Beex, L.A. Kiemeney, M.J.L. Ligtenberg, L.F. Massuger, C. Boetes, \\ P. Manders, and H.G. Brunner
}

\begin{abstract}
Purpose: Women with a hereditary predisposition for breast cancer have an extremely high risk of developing invasive breast carcinoma, and many women consider prophylactic mastectomy to avoid this risk. The use of prophylactic mastectomy is still debated. Identification of frequent premalignant lesions in mastectomy specimens would support the preventive concept of prophylactic mastectomy.

Patients and Methods: We performed a prospective study of breast specimens from 67 women at extremely high genetic risk of breast cancer, with or without previous breast cancer, who were undergoing prophylactic mastectomy $(66 \%$ were carriers of a BRCA1 or BRCA2 mutation). Breast specimens were studied by radiographic and macroscopic examination of 5-mm tissue slices, with subsequent histology of suspicious lesions and random samples from each quadrant of the breast and the nipple area.
\end{abstract}

$\mathrm{T}^{\mathrm{H}}$ HE DECISION regarding whether and when to undergo prophylactic mastectomy for hereditary breast cancer prevention is quite complex. A particular subject of debate is the utility of masectomy for breast cancer prevention. Some studies have stressed the paradox of performing more extensive surgery for breast cancer prevention than for actual disease. ${ }^{1-3}$ The gain in life expectancy may not be large, ${ }^{4,5}$ and the effects of prophylactic mastectomy on quality of life are not precisely known. ${ }^{6,7}$ A decision model estimated that the gain in life expectancy for a 30 -year-old woman who carries a BRCAl or $B R C A 2$ mutation from bilateral prophylactic mastectomy is 3 to 5 years. ${ }^{5}$ Recent studies support the concept that mastectomy is effective for breast cancer prevention in women at high hereditary risk of breast cancer. ${ }^{8,9}$ The effects of chemopreventive drugs, like tamoxifen, for the prevention of hereditary breast cancer are uncertain. ${ }^{10}$ The expected efficacy is low, ${ }^{11}$ especially for women carrying a $B R C A 1$ mutation, because most invasive breast cancers in $B R C A l$ mutation carriers are estrogen receptor-negative. ${ }^{12}$

Little is known about the early stages of breast cancer development in inherited forms of the disease. For instance, it is not known whether women with a hereditary risk of breast cancer are prone to develop high-risk histopathological lesions. Identification of premalignant lesions in prophylactically removed breasts would add plausibility to the concept of breast cancer risk reduction by prophylactic mastectomy in women at high hereditary risk of breast cancer.

We performed a prospective study on prophylactic mastectomy specimens from women with a hereditary predisposition for breast cancer (ie, women with a $30 \%$ to $85 \%$ lifetime risk of developing breast cancer). The aim of this study was to assess whether women at high hereditary risk for breast cancer have high-risk histopatho-
Results: In $57 \%$ of the women, one or more different types of high-risk histopathologic lesions were present: $37 \%$ atypical lobular hyperplasia, $39 \%$ atypical ductal hyperplasia, $25 \%$ lobular carcinoma-in-situ, and $15 \%$ ductal carcinoma-in-situ. A 4-mm invasive ductal carcinoma was found in one woman with ductal carcinoma-in-situ. None of these lesions was detected at palpation or mammography, which were performed before the mastectomy. The presence of high-risk lesions was independently related to age older than 40 years (odds ratio, 6.6; $P=.01$ ) and to bilateral oophorectomy before prophylactic mastectomy (odds ratio, 0.2; $P=0.02$ ).

Conclusion: Many women at high risk of hereditary breast cancer develop high-risk histopathologic lesions, especially after the age of $\mathbf{4 0}$ years. Surveillance does not detect such high-risk histopathologic lesions.

$J$ Clin Oncol 21:41-45. () 2003 by American Society of Clinical Oncology.

logic lesions and to determine the variables related to, and predictive for, the presence of such high-risk lesions.

\section{PATIENTS AND METHODS}

\section{Patients}

Prophylactic mastectomy was performed between 1989 and 2001 in women, with and without previous breast cancer, who were at high hereditary risk. Prophylactic mastectomy of the contralateral breast was performed in women who had previous breast cancer. Bilateral mastectomy was performed in women who did not have previous breast cancer but were at high genetic risk. Individuals were included who had been tested for germline BRCA1 or BRCA2 mutations that were associated with breast and/or ovarian cancer in their families. Six women were included because of familial clustering of breast cancer, without testing the presence of a $B R C A$ mutation. The lifetime risk of breast cancer in $B R C A$ mutation carriers is $55 \%$ to $85 \%,{ }^{13}$ and the lifetime risk of breast cancer in the group of hereditary breast cancer patients (patients whose breast cancer is not a result of $B R C A 1$ or $B R C A 2$ ) is at least $30 \%$, based on the model of Claus et al. ${ }^{14}$ Medical records were reviewed for family history, breast cancer-related risk factors (age, oophorectomy, previous breast cancer, menarche, duration of oral contraceptives, age at first pregnancy, and parity), physical examinations, and radiological examinations.

From the Hereditary Cancer Clinic, Departments of Human Genetics, Medical Oncology, Pathology, Surgery, Epidemiology and Biostatistics, Gynecology, and Radiology, University Medical Center Nijmegen, the Netherlands.

Submitted February 26, 2002; accepted August 26, 2002.

Both N. Hoogerbrugge and P. Bult contributed equally to this work. Address reprint requests to $N$. Hoogerbrugge, MD, Department of Human Genetics, University Medical Center Nijmegen, PO Box 9101, 6500 HB Nijmegen, the Netherlands; email: n.hoogerbrugge@antrg.azn.nl.

(C) 2003 by American Society of Clinical Oncology.

$0732-183 X / 03 / 2101-41 / \$ 20.00$ 


\section{Specimens}

The handling of 65 of 67 specimens was based on the correlated radiographic and pathologic technique developed by Egan, ${ }^{15}$ which has been routinely performed in our pathology department for many years. The method is described in detail elsewhere. ${ }^{16}$ The specimens were cooled and sliced in serial sections with approximately 5-mm intervals. Radiographs were made from the tissue slices. Suspicious lesions and randomly selected areas from each quadrant and the nipple were sampled, with a mean number of 19 samples per specimen. A review of the pathology report, histologic slides, and the simple mastectomy specimen radiographs was conducted. Atypical lobular hyperplasia (ALH), atypical ductal hyperplasia (ADH), and lobular carcinoma-in-situ (LCIS) were classified according to the criteria of Page et al. ${ }^{17,18}$ Ductal carcinoma-in-situ (DCIS) was classified according to the criteria of Holland et al. ${ }^{19}$

\section{Statistical Analysis}

Continuous variables were compared by $t$ tests, categorical variables, with the use of cross tables and Pearson's $\chi^{2}$ test. Only one breast from each patient was taken into evaluation. To evaluate whether it is possible to predict histologic abnormalities with a combination of baseline characteristics, we performed multivariate logistic regression analyses. In these analyses, histopathology was defined as the dependent variable. Odds ratios, with their $95 \%$ confidence intervals, were calculated as a measure of the predictive power of each characteristic, independent of the other characteristics in the model. Based on the outcome of the multivariate analysis, we calculated the probability of histologic abnormalities for each woman. Subsequently, these probabilities were used to construct a receiver operating characteristic (ROC) curve to visualize the predictive power of the combination of the variables. The area under the ROC curve quantified this predictive power.

\section{RESULTS}

\section{Patient Characteristics}

Two groups of women were studied. Women in one group had a unilateral prophylactic mastectomy, contralateral to a previous breast cancer $(n=26)$, with a mean time interval between breast cancer diagnosis and prophylactic mastectomy of $1 \pm 4$ years. Women in the second group had a bilateral prophylactic mastectomy, without previous breast cancer $(n=41)$. Chemopre- ventive drugs such as tamoxifen were not used by any of the women included. High-risk histopathologic lesions were found in both breasts of 13 of 41 women who had undergone bilateral mastectomy (aged 27 to 52 years; six BRCA mutation carriers). In seven of 41 women, only one breast was affected, and in 21 of 41 women, neither breast had high-risk lesions. High-risk lesions were found in 18 of 26 women who had undergone unilateral mastectomy. In case of bilateral mastectomy, the breast with the most severe lesions was taken into evaluation. The mean age of the women with bilateral prophylactic mastectomy was significantly lower than in the group of women with previous breast cancer who underwent unilateral prophylactic mastectomy ( $37 \pm 7$ years and $45 \pm 9$ years, respectively; $P<.001)$. There were significantly more $B R C A$ mutation carriers in the group of women who had undergone bilateral prophylactic mastectomy than in the group of women who had undergone unilateral prophylactic mastectomy $(80 \%$ and $42 \%$, respectively; $P=.01$ ). Other risk factors (Table 1 ) were not significantly different between the groups with bilateral or unilateral mastectomy (data not shown). Prophylactic oophorectomies were only performed in the group of women carrying a $B R C A$ mutation.

Table 1 summarizes the baseline characteristics of women who underwent prophylactic mastectomy. In 44 of 67 women, a $B R C A$ mutation was found (38 BRCAl and six BRCA2 mutations). To exclude overt malignancy, palpation was performed by a skilled practitioner the day before mastectomy, mammography was done in all patients ( $3 \pm 3$ months before the mastectomy), and 27 of 67 patients underwent magnetic resonance imaging (MRI) of the breasts ( $3 \pm 2$ months previous to the mastectomy). In all women, palpation, mammography, and MRI were without signs of breast cancer. In Table 1 , the characteristics of the groups, with and without high-risk histopathologic lesions, are listed. Compared with patients without high-risk lesions, patients with high-risk lesions

Table 1. Prevalence of Breast Cancer-Related Risk Factors in Patients Undergoing Prophylactic Mastectomy With and Without High-Risk Histopathologic Lesions

\begin{tabular}{lcccc}
\hline & $\mathrm{N}=67(\%)$ & $\begin{array}{c}\text { No High-Risk } \\
\text { Lesions, } \mathrm{n}= \\
29(\%)\end{array}$ & $\begin{array}{c}\text { High-Risk } \\
\text { Lesions, } \mathrm{n}=38 \\
(\%)\end{array}$ & $P^{*}$ \\
\hline Genetic risk factors & & & & \\
BRCA mutation carrier & $44(66)$ & $25(86)$ & $19(50)$ & .002 \\
Youngest family member breast cancer (years) & $38 \pm 10$ & $37 \pm 10$ & $39 \pm 10$ & $\mathrm{~ns}(.49)$ \\
Youngest family member ovarian cancer (years) & $51 \pm 7$ & $51 \pm 8$ & $51 \pm 6$ & $\mathrm{~ns}(.92)$ \\
Nongenetic risk factors & & & & \\
Age at prophylactic mastectomy (years) & $39 \pm 8$ & $37 \pm 7$ & $43 \pm 11$ & .008 \\
Prophylactic oophorectomy & $29(43)$ & $17(58)$ & $12(32)$ & .030 \\
Oophorectomy before mastectomy & $17(25)$ & $10(34)$ & $7(18)$ & $\mathrm{ns}(.13)$ \\
Previous breast cancer & $26(38)$ & $8(28)$ & $18(47)$ & $\mathrm{ns}(.10)$ \\
Age at previous breast cancer (years) & $42 \pm 10$ & $35 \pm 5$ & $47 \pm 9$ & .002 \\
Age at menarche (years) & $13.0 \pm 1.5$ & $13.4 \pm 1.4$ & $12.9 \pm 1.6$ & $\mathrm{~ns}(.23)$ \\
Parity & $2.1 \pm 1.2$ & $2.2 \pm 1.1$ & $1.9 \pm 1.3$ & $\mathrm{~ns}(.43)$ \\
Age at first pregnancy (years) & $27.0 \pm 4.1$ & $26.9 \pm 4.7$ & $27.3 \pm 3.5$ & $\mathrm{~ns}(.31)$ \\
Duration of oral contraceptive (years) & $9.2 \pm 6.2$ & $9.7 \pm 7.0$ & $8.8 \pm 7.3$ & $\mathrm{~ns}(.73)$ \\
Body mass index (kg/m ${ }^{2}$ ) & $23.8 \pm 5.3$ & $24.0 \pm 4.3$ & $23.1 \pm 6.4$ & $\mathrm{~ns}(.53)$ \\
Breast specimen weight (g) & $567 \pm 338$ & $551 \pm 342$ & $590 \pm 354$ & $\mathrm{~ns}(.66)$ \\
Nodular breasts at palpation & $13(19)$ & $4(14)$ & $9(24)$ & $\mathrm{ns}(.24)$ \\
Difficult interpretable mammography & $23(34)$ & $11(38)$ & $12(32)$ & $\mathrm{ns}(.66)$ \\
\hline NOTE
\end{tabular}

NOTE.

Abbreviation: ns, not significant.

Data are presented as mean $\pm S D$, or as number with the percentage between brackets.

${ }^{*}$ Indicates the significance of the difference between the group with and without high-risk lesions.

Downloaded from ascopubs.org by Radboud University Nijmegen on January 21, 2019 from 131.174.248.154 Copyright (๑) 2019 American Society of Clinical Oncology. All rights reserved. 
were, on average, 6 years older $(P<.01)$, less likely to have had a previous oophorectomy ( $18 \% \vee 34 \%$, respectively; $P<.05)$, and had less chance of carrying a BRCA mutation $(50 \% \vee 86 \%$, respectively; $P<.01$ ).

\section{Histopathologic Findings}

Table 2 summarizes the histopathologic findings. One or more high-risk lesions was found in $57 \%$ of the patients, with ALH in $37 \%$, ADH in $39 \%$, LCIS in $25 \%$, and DCIS in $15 \%$ of the women. Table 2, which also gives the histopathologic findings and main risk factors of women younger and older than 40 years of age, shows that there were significantly more lesions in the group aged 40 years and older.

Table 3 presents the results of the multivariate logistic regression analysis. Age older than 40 years and oophorectomy had a significant predictive value for the presence or absence of histologic abnormalities in the mastectomy specimens. Previous breast cancer did not add any predictive value. This multivariate regression analysis was also performed separately for the groups with and without previous breast cancer, giving comparable predictive values (data not shown). The presence of $B R C A 1$ or BRCA2 mutations was not included in the model, because this variable was highly correlated with oophorectomy. We used the model to calculate the probability for each woman to have histologic abnormalities. Subsequently, we considered this probability as a diagnostic test for histologic abnormalities, and we evaluated its predictive value with an ROC curve. The area under the curve was 0.72 (95\% confidence interval, 0.58 to 0.83 ). Thus, age and previous oophorectomy have limited predictive ability that is not sufficient to predict abnormalities with any certainty. This is also demonstrated in Table 4, which presents the distribution of patients according to these characteristics. High-risk lesions were present at appreciable frequency in all subgroups; however, some clear differences were evident. High-risk histopathologic lesions were detected in almost 50\% (16 of 37 women) of the women younger than age 40 years. Among $29 \mathrm{BRCA}$ mutation carriers, only one of five women had high-risk lesions after oophorectomy, compared with 10 of 24 women without previous oophorectomy. Similarly, five of eight women whose families were negative for $B R C A$ mutations had high-risk lesions. None of them had undergone an oophorectomy. A higher frequency of lesions and the same effect of oophorectomy was observed in women older than age 40 years. Among women from families with strong genetic predisposition but with no demonstrable $B R C A$ mutation, almost all of them (14 of 15 women) had at least one

Table 2. Histopathologic Findings and Relevant Risk Indicators of Patients Younger Versus Older Than 40 Years of Age

\begin{tabular}{lcccc}
\hline & $N=67(\%)$ & $\begin{array}{c}<0 \text { years, } \\
n=37(\%)\end{array}$ & $\begin{array}{c}\geq 0 \text { years, } \\
n=30(\%)\end{array}$ & $P^{*}$ \\
\hline Overall presence of high-risk lesions & $38(57)$ & $16(43)$ & $22(73)$ & .01 \\
ALH & $25(37)$ & $8(22)$ & $17(57)$ & .003 \\
ADH & $26(39)$ & $10(27)$ & $16(53)$ & .03 \\
LCIS & $17(25)$ & $4(11)$ & $13(43)$ & .002 \\
DCIS & $10(15)$ & $3(8)$ & $7(23)$ & .08 \\
Oophorectomy before mastectomy & $17(25)$ & $5(14)$ & $12(40)$ & .013 \\
BRCA mutation carrier & $44(66)$ & $29(78)$ & $15(50)$ & .015 \\
Previous breast cancer & $26(39)$ & $7(19)$ & $19(63)$ & .000 \\
\hline
\end{tabular}

NOTE.

Data are presented as the actual number, with the percentage between brackets.

*Indicates the significance of the difference between the group older and younger than 40 years.
Table 3. Multivariate Regression Analysis of Factors Predicting the Presence of High-Risk Pathologic Findings

\begin{tabular}{lccc}
\hline & Odds Ratio & $95 \% \mathrm{Cl}$ & $P$ \\
\hline Age $\geq 40$ years & 6.6 & $1.5-28.6$ & .011 \\
Oophorectomy before mastectomy & 0.2 & $0.04-0.8$ & .02 \\
Previous breast cancer & 1.2 & $0.4-4.2$ & .7 \\
\hline
\end{tabular}

Abbreviation: $\mathrm{Cl}$, confidence interval.

high-risk histopathologic lesion on careful examination of the mammary gland. None of these women had undergone an oophorectomy. In contrast, the majority of older $B R C A$ mutation carriers (12 of 15 women) had undergone an oophorectomy. In this group, only $50 \%$ were found to have high-risk lesions. These data confirm that age and oophorectomy (or possibly BRCA mutation status) influence the risk of having high-risk histopathologic lesions of the mammary gland.

Ten women had DCIS at the time of prophylactic mastectomy. These women were of special interest because DCIS has an extremely high risk of subsequent invasive cancer. Two of the women had undergone an oophorectomy. The mean age of the group of women with DCIS was 45 years (range, 29 to 62 years). DCIS was moderate or high grade in seven of 10 women, with a mean size of $9 \mathrm{~mm}$ (range, 2 to $40 \mathrm{~mm}$ ). Four of 10 women carried a BRCA mutation, and $50 \%$ had previous breast cancer. Clinical breast examination was performed in all of these women the day before mastectomy. Mammography was done, on average, 3 months before the mastectomy (maximum, 6 months). Clinical breast examinations and mammograms did not disclose any signs of malignancy. In four of 10 patients, mammography was combined with MRI, which was also unremarkable. In seven of 10 women with DCIS, microcalcifications were visible on postsurgery x-rays of the specimen, and in two cases, these microcalcifications led to the diagnosis of DCIS. ADH, ALH, or LCIS was present in most of these women with DCIS. A 4-mm invasive ductal carcinoma was found in one 52-year-old woman with DCIS who underwent a bilateral prophylactic mastectomy. In retrospect, this invasive carcinoma was not identified on mammography or MRI of the breast, which was performed 2 months before the mastectomy. In addition to this invasive carcinoma and DCIS, both breasts showed ADH and ALH, and LCIS was diagnosed in the contralateral breast.

\section{DISCUSSION}

Women with a hereditary predisposition for breast cancer are prone to develop high-risk lesions in their breasts. In our study, these lesions included ADH, ALH, LCIS, or DCIS, and they were present in $73 \%$ of the women aged 40 years and older. The high prevalence of lesions in our study may be explained by a careful macroscopic examination of the breast specimens combined with specimen radiograms and a large number of excisions. This combined procedure made possible the detection of small foci of microcalcifications or small distortions, and it allowed the detection of normally occult lesions such as carcinoma-in-situ or atypical hyperplasia.

High-risk histopathologic lesions were found in both breasts of 13 of 41 women who had undergone mastectomy. In seven of 41 women, one breast was affected, and in 21 of 41 women, neither breast had high-risk lesions. It is therefore clear that development of high-risk lesions tended to occur simultaneously Downloaded from ascopubs.org by Radboud University Nijmegen on January 21, 2019 from 131.174.248.154 Copyright @ 2019 American Society of Clinical Oncology. All rights reserved. 
Table 4. Distribution of Patients With and Without High-Risk Histopathologic Lesions, According to Age,

BRCA Mutation, Previous Breast Cancer, and Previous Oophorectomy

\begin{tabular}{|c|c|c|c|c|c|c|c|c|}
\hline & \multicolumn{4}{|c|}{ High-Risk Lesions } & \multicolumn{4}{|c|}{ No High-Risk Lesions } \\
\hline & \multicolumn{2}{|c|}{ Age $<40$ Years } & \multicolumn{2}{|c|}{ Age $\geq 40$ Years } & \multicolumn{2}{|c|}{ Age $<40$ Years } & \multicolumn{2}{|c|}{ Age $\geq 40$ Years } \\
\hline & $B R C A^{+}$ & $B R C A^{-}$ & $B R C A^{+}$ & $B R C A^{-}$ & $B R C A^{+}$ & $B R C A^{-}$ & $B R C A^{+}$ & $B R C A^{-}$ \\
\hline \multicolumn{9}{|l|}{ Previous breast cancer } \\
\hline Oophorectomy & 0 & 0 & 4 & 0 & 1 & 0 & 2 & 0 \\
\hline No oophorectomy & 1 & 1 & 1 & 11 & 2 & 2 & 0 & 1 \\
\hline \multicolumn{9}{|c|}{ No previous breast cancer } \\
\hline Oophorectomy & 1 & 0 & 2 & 0 & 3 & 0 & 4 & 0 \\
\hline No oophorectomy & 9 & 4 & 1 & 3 & 12 & 1 & 1 & 0 \\
\hline
\end{tabular}

in both breasts. Moreover, even within a single breast, there was a strong tendency for multiple lesions to occur. Thus, one would expect that high-risk lesions should be particularly frequent in women with a previous history of cancer of the contralateral breast. This was indeed the case with high-risk lesions, which were present in 18 of 26 breast specimens examined. These data indicate that although the genetic factor determines high relative risk, the actual occurrence of high-risk histopathologic lesions is a result of other systemic factors that are unrelated to the gene. We examined factors that might influence the development or detection of high-risk lesions, such as age, previous breast cancer, and previous oophorectomy. Of these factors, only age correlated positively, as would be expected. However, there was a strong negative correlation between the occurrence of high-risk lesions and previous oophorectomy. We cannot exclude that the inverse relation between high-risk lesions and previous oophorectomy was caused by selection, as all women who underwent a prophylactic oophorectomy were $B R C A$ mutation carriers. The presence of a $B R C A$ mutation was significantly lower in the group with high-risk lesions than in the group without high-risk lesions. The lower prevalence of high-risk lesions in women who carried a $B R C A$ mutation may indicate a different pathophysiology in the progression of precancerous lesions to invasive cancer than in the group without such a mutation. A relation between oophorectomy and a decreased breast cancer risk is supported by Rebbeck and colleagues, who showed that breast cancer risk was reduced by almost 50\% after bilateral prophylactic oophorectomy in BRCAl mutation carriers. These authors speculated that the decreased production of sex hormones after oophorectomy is responsible for the reduction in breast cancer risk. ${ }^{20}$ Turner et $\mathrm{al}^{21}$ showed that relapses of breast cancer in BRCA mutation carriers were mostly new primary tumors, although in the general population, relapses were most often recurrences of the previous tumor. This supports our findings of frequent synchronous and metachronous high-risk lesions in women who are at high hereditary risk for breast cancer. In addition, we found these lesions to exist more frequently in high-risk women without a $B R C A$ mutation than in women with a BRCA mutation. This indicates that all women at high hereditary risk for breast cancer are prone to develop multiple lesions, independent of whether they do or do not carry a BRCA mutation.

DCIS was present in 10 of 67 women. This finding is of great concern because, as in the general population, unresected DCIS strongly increases the risk of subsequent invasive breast cancer from $30 \%$ to $50 \%$ after 10 years. ${ }^{22}$ Therefore, DCIS should be resected completely to prevent invasive breast cancer. In our study, DCIS was not detected by either palpation or mammography before mastectomy. In four of the 10 patients with DCIS, MRI of the breasts was performed, which also failed to detect the DCIS lesions. Most women with DCIS were older than 40 years of age. Although the occurrence of high-risk lesions, such as DCIS, was strongly related to age and correlated negatively to previous oophorectomy, the predictive value of these variables was not sufficient to predict abnormalities with any certainty. Therefore, age and oophorectomy cannot be used to counsel women who are at high hereditary risk about whether or when to perform prophylactic mastectomy. We concluded that clinical breast examination, mammography, and breast cancer risk factors are insufficient to predict the presence of high-risk histopathologic lesions.

In addition to regular surveillance, current risk reduction strategies for women at hereditary risk for breast cancer include prophylactic mastectomy, oophorectomy (or both), and chemoprevention. The effects of tamoxifen, currently the most important chemopreventive drug, have been questioned with respect to its efficacy for $B R C A 1$ mutation carriers. ${ }^{11,12}$ Breast cancers that arise in carriers of $B R C A 1$ mutations are commonly estrogen receptor-negative, unlike tumors associated with $B R C A 2$ mutations, indicating that the effect of tamoxifen might be selective for distinct genotypes. The results of our study support the concept of early prophylactic mastectomy or prophylactic oophorectomy to reduce the genetic risk of breast cancer.

The fact that an occult carcinoma was present in only one of 67 patients in our study might indicate that surveillance is as effective as prophylactic mastectomy. However, in our study, all 10 DCIS cases were missed by surveillance, and it was recently shown by Meijers-Heijboer at $\mathrm{al}^{9}$ that surveillance is less effective than prophylactic mastectomy in preventing breast cancer deaths.

This study lacks a control group of women without hereditary risk. This is because women who undergo an operation for breast reduction have only part of their breasts removed, and these women, for the most part, belong to a younger age group. Fortunately, healthy women who are of the same age group as our patients only rarely come for autopsy. Despite the absence of this control group, the high prevalence of DCIS and other high-risk lesions in the prophylactically removed breasts strongly supports the relevance of prophylactic mastectomy in women who are at high hereditary risk for breast cancer.

The occurrence of lesions found in the study population is much higher than that reported in the literature. ${ }^{23-27}$ In 25 women with a family history of breast cancer, Khurana et $\mathrm{al}^{23}$ reported $\mathrm{ADH}$ in $8 \%$, ALH in $4 \%$, and DCIS in $4 \%$ of the women. In the general population, ALH and $\mathrm{ADH}$ represent a relatively uncommon diagnosis, constituting less than $5 \%$ of all benign breast biopsies. ${ }^{17,28}$ Dupont et $\mathrm{al}^{29}$ showed that women with $\mathrm{ADH}$ or Nijmegen on January 21, 2019 from 131.174.248.154 Copyright (C) 2019 American Society of Clinical Oncology. All rights reserved. 
ALH and a family history of breast cancer have an 11-fold increased risk of developing breast cancer. The reported prevalence of LCIS in the general population is variable, ranging from $0.5 \%$ to $3.6 \%{ }^{30,31}$ Controversy exists with regard to the natural course of LCIS. Specifically, there is disagreement about whether LCIS is a precursor of invasive disease or merely a marker of subsequent invasive carcinoma risk. In women diagnosed with LCIS, approximately $30 \%$ will develop an invasive carcinoma,${ }^{32}$ most often of the ductal type. ${ }^{33}$ LCIS is most likely a risk indicator for breast cancer, but it is not itself a true precursor for invasive disease in most patients.

For women carrying a BRCA mutation, the risk of breast cancer begins to increase before the age of 25 years, with a steep increase after age 40 years. The cumulative risk of developing breast cancer before the age of 40 years is approximately $15 \%$, whereas the risk of developing breast cancer before the age of 50 years is $40 \%$ to $50 \% .^{13,34}$ In our study, all types of high-risk lesions showed a higher prevalence in the group of women aged
40 years and older. Multivariate regression analysis showed that age is independently related to the occurrence of high-risk lesions. This indicates that there is a time-dependent development of lesions, probably under the influence of genetic susceptibility, which precedes the occurrence of invasive breast cancer.

In conclusion, this study shows that the majority of women at high hereditary risk of breast cancer have high-risk histopathologic lesions in one or both breasts. Although not all lesions will develop into invasive carcinoma, they may predict the occurrence of subsequent invasive breast cancer elsewhere in the breast. The risk for developing (contralateral) invasive carcinoma is high for patients with hereditary predisposition. ${ }^{35}$ The high rate of high-risk lesions found in our study helps to explain this risk.

\section{ACKNOWLEDGMENT}

We thank J.H. van Krieken, MD, for his critical comments on the manuscript and R.F. van der Sluis, MD, for performing most of the prophylactic mastectomies.

\section{REFERENCES}

1. Klijn JG, Janin N, Cortes-Funes H, et al: Should prophylactic surgery be used in women with a high risk for breast cancer? Eur J Cancer 33:2149-2159, 1997

2. Eisen A, Weber BL: Prophylactic mastectomy-the price of fear. N Engl J Med 340:137-138, 1999

3. Fentiman IS: Prophylactic mastectomy: Deliverance or delusion. Br J Med 317:1402-1403, 1998

4. Schrag D, Kuntz KM, Garber JE, et al: Decision analysis: Effects of prophylactic mastectomy and oophorectomy on life expectancy among women with BRCA1 or BRCA2 mutations. N Engl J Med 336:1465-1471, 1997

5. Schrag D, Kuntz KM, Garber JE, et al: Life expectancy gains from cancer prevention strategies for women with breast cancer and BRCA1 or BRCA2 mutations. JAMA 283:617-624, 2000

6. Frost MH, Schaid DJ, Sellers TA, et al: Long-term satisfaction and psychological and social function following bilateral prophylactic mastectomy. JAMA 284:319-324, 2000

7. Hughes KS, Papa MZ, Whitney T, et al: Prophylactic mastectomy and inherited predisposition to breast carcinoma. Cancer 86:1682-1696, 1999

8. Hartmann LC, Schaid DJ, Woods JE, et al: Efficacy of bilateral prophylactic mastectomy in women with family history of breast cancer. N Engl J Med 340:77-84, 1999

9. Meijers-Heijboer H, van Geel B, van Putten WLJ, et al: Breast cancer after prophylactic bilateral mastectomy in women with a BRCA1 or BRCA2 mutation. N Engl J Med 345:159-164, 2001

10. Narod SA: Hormonal prevention of hereditary breast cancer. Ann N Y Acad Sci 952:36-43, 2001

11. King MC, Wieand S, Jale K, et al: National Breast and Bowel Project NSABP-P breast cancer prevention trial. JAMA 286:2251-2256, 2001

12. Lakhani SR, Van De Vijver MJ, Jacquemier J, et al: The pathology of familial breast cancer: Predictive value of immunohistochemical markers estrogen receptor, progesterone receptor, HER-2, and p53 in patients with mutations in BRCA1 and BRCA2. J Clin Oncol 20:2310-2318, 2002

13. Ford D, Easton DF, Stratton M, et al: Genetic heterogeneity and penetrance analysis of BRCA1 and BRCA2 genes in breast cancer families. The Breast Cancer Linkage Consortium. Am J Hum Genet 62:676-689, 1998

14. Claus EB, Risch N, Thompson WD: Autosomal dominant inheritance of early-onset breast cancer. Implications for risk prediction. Cancer 73:643651,1994

15. Egan RL: Multicentric breast carcinomas: Clinical-radiographic-pathologic whole organ studies and 10-year survival. Cancer 49:1123-1130, 1982

16. Holland R, Veiling SH, Mravunac M, et al: Pathologic multifocality of Tis, T1-2 breast carcinomas. Implications for clinical trials of breastconserving surgery. Cancer 56:979-990, 1985

17. Page DL, Dupont WD, Rogers LW, et al: Atypical hyperplastic lesions of the female breast. A long term follow-up study. Cancer 55:26982708, 1985
18. Page DL, Rogers LW: Combined histologic and cytologic criteria for the diagnosis of mammary atypical ductal hyperplasia. Hum Pathol 23:10951097, 1992

19. Holland R, Peterse JL, Millis RR, et al: Ductal carcinoma-in-situ: A proposal for a new classification. Semin Diagn Path 11:167-180, 1994

20. Rebbeck TR, Levin AM, Eisen A, et al: Breast cancer risk after bilateral prophylactic oophorectomy in BRCA1 mutation carriers. J Natl Cancer Inst 91:1475-1479, 1999

21. Turner BC, Harrold E, Matloff E, et al: BRCA1/BRCA2 germline mutations in locally recurrent breast cancer patients after lumpectomy and radiation therapy: Implication for breast-conserving management in patients with BRCA1/BRCA2 mutations. J Clin Oncol 17:3017-3024, 1999

22. Frykberg ER, Bland KI: In situ breast carcinoma. Adv Surg 26:29-72, 1993

23. Khurana KK, Loosmann A, Numann PJ, et al: Prophylactic mastectomy. Pathologic findings in high-risk patients. Arch Pathol Lab Med 124:378-381, 2000

24. Karabela-Bouropoullou V, Liapi-Avgeri G, Iliopoulou E, et al: Histological findings in breast tissue specimens from reduction mammoplasties. Pathol Res Pract 190:792-798, 1994

25. Alpers CE, Wellings SR: The prevalence of carcinoma-in-situ in normal and cancer associated breasts: Hum Pathol 16:796-807, 1985

26. Ringberg A, Palmer B, Linell F, et al: Bilateral and multifocal breast carcinoma: A clinical and autopsy study with special emphasis on carcinoma-in-situ. Eur J Surg Cancer 17:20-29, 1991

27. Roubidoux MA, Helvie MA, Wilson TE, et al: Women with breast cancer: Histologic findings in the contralateral breast. Radiology 203:691-694, 1997

28. Osborne MP, Borgen PI: Atypical ductal and lobular hyperplasia and breast cancer risk. Surg Oncol Clin N Am 2:1-11, 1993

29. Dupont WD, Page DL: Risk factors for breast cancer in women with proliferative breast disease. N Engl J Med 312:146-151, 1985

30. Abner AA, Connolly JL, Recht A, et al: The relation between the presence and extent of lobular carcinoma-in-situ and the risk of local recurrence for patients with infiltrating carcinoma of the breast treated with conservative surgery and radiation therapy. Cancer 88:1072-1077, 2000

31. Singletary SE: Lobular carcinoma-in-situ of the breast: 31 year experience at the University of Texas M.D. Anderson Cancer Center. Breast Dis 7:157-163, 1994

32. Hutter RV: The management of patients with lobular carcinoma-insitu of the breast. Cancer 53:798-802, 1984

33. Rosen PP, Braun DW, Kinne DW: The clinical significance of pre-invasive breast carcinoma. Cancer 46:35-51, 1980

34. Easton DF, Ford D, Bishop DT: The Breast Cancer Linkage Consortium. Breast and ovarian incidence in BRCA1 mutation carriers. Am J Hum Genet 56:265-271, 1995

35. Verhoog LC, Brekelmans CTM, Seynaeve C, et al: Contralateral breast cancer risk is influenced by the age of onset in BRCA1-associated breast cancer. Br J Cancer 83:384-386, 2000 